Palavras chave: Restrição nos parâmetros inferência bayesiana mensuração florestal

Histórico:

Recebido 14/10/2013

Aceito 13/04/2015

Keyworks: restriction parameters Bayesian inference forest measurement

Correspondence: engagri@yahoo.com.br
Monica Fabiana Bento Moreira', Cláudio Roberto Thiersch', Marinho Gomes de Andrade², José Roberto Soares Scolforo'

\section{ESTIMATIVA DA RELAÇÃO HIPSOMÉTRICA COM MODELOS NÃO LINEARES AJUSTADOS POR MÉTODOS BAYESIANOS EMPÍRICOS}

RESUMO: Neste trabalho, está sendo proposta uma abordagem bayesiana para resolver o problema de inferência com restrições nos parâmetros, em modelos não lineares que representam a relação hipsométrica em clones de Eucalyptus sp. As estimativas Bayesianas são calculadas com a técnica de simulação de Monte Carlo em Cadeia de Markov (MCMC). O método proposto foi aplicado a diferentes conjuntos de dados reais, dos quais foram selecionados dois para mostrar os resultados obtidos. Estes foram comparados aos obtidos pelo método de mínimos quadrados, destacando-se a superioridade da abordagem Bayesiana empírica proposta, uma vez que esta abordagem sempre gera resultados coerentes biologicamente para a relação hipsométrica.

\section{ESTIMATE OF THE HYPSOMETRIC RELATIONSHIP WITH NONLINEAR MODELS FITTED BY EMPIRICAL BAYESIAN METHODS}

ABSTRACT: In this paper we propose a Bayesian approach to solve the inference problem with restriction on parameters, regarding to nonlinear models used to represent the hypsometric relationship in clones of Eucalyptus sp. The Bayesian estimates are calculated using Monte Carlo Markov Chain (MCMC) method. The proposed method was applied to different groups of actual data from which two were selected to show the results. These results were compared to the results achieved by the minimum square method, highlighting the superiority of the Bayesian approach, since this approach always generate the biologically consistent results for hipsometric relationship. 


\section{INTRODUÇÃO}

A quantificação da altura das árvores é fundamental principalmente para o cálculo do volume de madeira dos povoamentos e para se fazer classificações destes quanto às suas produtividades. A estimação indireta da altura da árvore é uma prática comum em inventários florestais, uma vez que a medição de todas as árvores em pé através de hipsômetros é uma operação onerosa e sujeita a erros.

Neste sentido, tem-se como prática medir na parcela a altura de parte das árvores e o diâmetro de todas e, através dos pares altura-diâmetro mensurados, estabelecer uma relação matemática que possibilite estimar as alturas das demais árvores contidas na parcela. Essas relações matemáticas são conhecidas como equações hipsométricas, que buscam estimar a altura das árvores através da relação DAP (Diâmetro do tronco da árvore a $1,3 m$ do solo) e $H$ (altura total da árvore). Essa técnica de mensuração na qual é medido o diâmetro de todas as árvores da parcela e a altura total de parte delas, resulta em uma sensível redução nos custos do inventário florestal e em uma operacionalização mais eficaz (RIBEIRO et al., 20I0).

Buscando melhorar as estimativas das alturas, os métodos e modelos adotados têm-se tornado cada vez mais sofisticados (SOARES et al., 2004). Por sua vez, existe uma dificuldade na escolha do melhor modelo para representar estas relações, devido a não linearidade da relação entre as variáveis envolvidas e das restrições impostas aos parâmetros dos modelos, por razões biológicas. Este fato torna o ajuste de modelos matemáticos para representar as relações hipsométricas um problema de regressão não linear com restrições nos parâmetros. A abordagem desse problema com os métodos convencionais de inferência estatística, tais como método de máxima verossimilhança e o método de mínimos quadrados torna-se ineficiente para vários modelos propostos. Uma abordagem bayesiana mostra-se como uma alternativa promissora por permitir incorporar, a priori, restrições biológicas aos parâmetros dos modelos considerados (THIERSCH et. al., 20I3).

Existe uma profusão de modelos que descrevem essa relação (SOARES et al., 2004). Não foi foco deste trabalho questionar os modelos hipsométricos mais utilizados no setor florestal, mas tentar estimar de forma mais precisa e eficiente seus parâmetros com amostras de tamanho reduzido utilizando uma abordagem Bayesiana.

\section{MATERIAL E MÉTODOS}

\section{Localização e caracterização da área}

Os dados utilizados para testar a metodologia proposta foram coletados em florestas plantadas de clones de Eucalyptus sp., da Fibria S.A., na região de Itapetininga, ao sul do Estado de São Paulo, com coordenadas geográficas $23^{\circ} 56^{\prime} \mathrm{S}$ de latitude e $42^{\circ} 2 \mathrm{I}^{\prime} \mathrm{W}$ de longitude, a uma altitude média de $655 \mathrm{~m}$.

\section{Amostragem}

Foram utilizadas cerca de 1000 parcelas circulares e com área de $500 \mathrm{~m}^{2}$ locadas em povoamentos com idades variando de 2 a 8 anos, das quais foram selecionadas duas, com e sem coerência biológica, para exemplificar os resultados obtidos. Sendo que, a parcela A foi mensurada em um povoamento de 4,34 anos de idade, espaçamento de 3,0×2,0m com I 4 fustes e a parcela $B$ em povoamento de 6,I2 anos, espaçamento de $3,0 \times 3,0 \mathrm{~m}$ e número de fustes igual a 15 .

\section{Modelos hipsométricos}

De forma geral pode-se representar a relação entre o diâmetro (DAP) do tronco da árvore e a altura total da árvore $(\mathrm{H})$ usando a relação (equação $\mathrm{I}$ ), sendo: em que $i=1, \ldots, \mathrm{n} ; y_{i}$ e $x_{i}$ são funções monotônicas da altura $\mathrm{H}_{\mathrm{i}}$ e do diâmetro $\mathrm{DAP}_{\mathrm{i}}$, respectivamente, $\in_{i}$ é um erro aleatório.

$y_{i}=\boldsymbol{\eta}\left(x_{i} ; \boldsymbol{\beta}\right)+\epsilon_{i}$

Dentre os diversos modelos hipsométricos utilizados no meio florestal, foram selecionados três, os quais podem ser vistos na Tabela 1.

Os parâmetros dos modelos apresentados na Tabela 1 estão sujeitos às restrições $\beta_{i}>0, i=0,1,2$, em que $\mathrm{H}$ representa a altura total da árvore e DAP, o diâmetro do tronco da árvore a uma altura de I,30m do solo. 
TABELA 1 Relação dos modelos de hipsometria.

TABLE 1 List of hypsometric models.

\begin{tabular}{lccc}
\hline \multicolumn{1}{c}{ Autor } & $y$ & $x$ & $\eta(x, \boldsymbol{\beta})$ \\
\hline Stoffel(1955) & $\ln (H)$ & $\ln (D A P)$ & $\beta_{0}+\beta_{1} x$ \\
Petterson(Curtis, 1967) & $\ln (H)$ & $1 / D A P$ & $-3 \ln \left(\beta_{0}+\beta_{1} x\right)$ \\
Prodan(1965, 1968) & $\ln (H)$ & $1 / D A P$ & $-\ln \left(\beta_{0} x^{2}+\beta_{1} x+\beta_{2}\right)$ \\
\hline
\end{tabular}

\section{Estimador de mínimos quadrados}

Neste trabalho, no lugar das observações pareadas $\left\{\left(\mathrm{H}_{1}, \mathrm{DAP}_{1}\right), \ldots,\left(\mathrm{H}_{\mathrm{n}}, \mathrm{DAP}_{\mathrm{n}}\right)\right\}$ foi utilizada a relação apropriada para cada modelo dado na Tabela 1 , e foram consideradas as observações transformadas $\left\{\left(y_{1}, x_{1}\right), \ldots,\left(y_{n}, x_{n}\right)\right\}$, onde $y_{i}=g_{1}\left(H_{i}\right)$ e $x_{i}=g_{1}\left(D A P_{i}\right)$, com $g_{1}($.$) e g_{2}($.$) dados na Tabela 1$.

Foi denotado por $\beta=\left(\beta_{0}, \ldots, \beta_{s}\right)^{\prime}$ o vetor de parâmetros de cada modelo e descrito de forma geral o modelo para as $n$ observações transformadas, na forma vetorial, (equação 2), sendo: $\mathrm{Y}=\left(\mathrm{y}_{1}, \ldots, \mathrm{y}_{\mathrm{n}}\right)^{\prime}, \mathrm{x}=\left(\mathrm{x}_{1}, \ldots, \mathrm{x}_{\mathrm{n}}\right)^{\prime}$, $\varepsilon=\left(\varepsilon_{1}, \ldots, \varepsilon_{n}\right)^{\prime}$ e $\boldsymbol{\eta}(\boldsymbol{x} ; \boldsymbol{\beta})=\left(\eta\left(x_{1} ; \boldsymbol{\beta}\right), \ldots, \eta\left(x_{n} ; \boldsymbol{\beta}\right)\right)^{\prime}$.

$\boldsymbol{Y}=\boldsymbol{\eta}(\boldsymbol{x} ; \boldsymbol{\beta})+\boldsymbol{\epsilon}$

A notação $\eta(\cdot ; \beta)$ foi utilizada para denotar que a função $\eta$ tem como parâmetro o vetor $\beta$. Portanto, o estimador de mínimos quadrados para o vetor de parâmetros $\beta$ foi calculado resolvendo-se o problema de minimização dado pela equação 3, sendo: onde $\mathrm{R}^{\mathrm{P}}$ é o espaço real $\mathrm{p}$ dimensional. Foi denotado por $\eta(x ; \hat{\beta})=\left(\eta\left(x_{1} ; \hat{\beta}\right), \ldots, \eta\left(x_{n} ; \hat{\beta}\right)\right)$ a avaliação da função $\eta(x ; \beta)$ quando os parâmetros $\beta$ foram substituídos por suas estimativas $\hat{\beta}$.

$\widehat{\beta}=\min _{\beta \in R^{p}}(\mathbf{Y}-\eta(\mathbf{x} ; \beta))^{\prime}(\mathbf{Y}-\eta(\mathbf{x} ; \beta))$

Então, um estimador não viciado [4] para a variância $\sigma^{2}$.

$\sigma^{2}=\frac{1}{n-1}(\mathbf{Y}-\eta(\mathbf{x} ; \widehat{\beta}))^{\prime}(\mathbf{Y}-\eta(\mathbf{x} ; \widehat{\beta}))$

Devido ao pequeno tamanho $n$ das amostras que geralmente são utilizadas em situações práticas, as estimativas de mínimos quadrados irrestritas para os parâmetros dadas em (2) resultam, em muitos casos, em valores inconsistentes biologicamente, ou seja, estimadores negativos que não permitem predizer os valores das alturas das árvores quando se considera árvores com diâmetro muito pequeno ou muito grande (modelos com parâmetros negativos são inconsistentes para os valores extremos). Para resolver este problema a solução foi a inclusão das restrições nos parâmetros, isso acarretou em (2) as restrições do tipo $\beta \in R$, onde $R=\left\{a_{i}<\beta_{i}<b_{i}, i=0, \ldots, p\right\}$. Na definição destas restrições foi considerado inclusive os casos em que alguns ou todos os $a_{i}$ podem ser $-\infty$ e os $b_{i}$ podem ser $+\infty$. A solução numérica deste problema pode ser encontrada, mas exige condições iniciais adequadas, caso contrário implica em muitas dificuldades de convergência.

Para contornar tal dificuldade, está sendo proposta uma abordagem Bayesiana para o cálculo dessas estimativas. Na abordagem Bayesiana as informações a priori sobre os parâmetros podem compensar parcialmente a pouca informação extraída somente dos dados quando estes constituem uma pequena amostra.

\section{Abordagem bayesiana}

A abordagem Bayesiana do problema de inferência dos parâmetros de um modelo assume que estes parâmetros são variáveis aleatórias e qualquer informação inicial sobre eles pode ser modelada por uma função densidade de probabilidade $a$ priori.

Combinando-se estas densidades a priori com a função de verossimilhança dos dados, através do teorema de Bayes, chega-se a função densidade de probabilidade a posteriori. Denotando-se o vetor de parâmetros de um modelo por $\theta$, a densidade a priori por $\pi_{0}(\theta)$ e a função de verossimilhança associada a um conjunto de observações $D$ por $L(\theta \mid D)$, então a função densidade de probabilidade a posteriori é dada pela equação 5 .

$\pi(\theta \mid \mathbf{D})=\frac{L(\theta \mid \mathbf{D}) \pi_{0}(\theta)}{\int_{\Theta} L(\theta \mid \mathbf{D}) \pi_{0}(\theta) d \theta}$

A integral em [5] é uma integral múltipla sobre o domínio de definição dos parâmetros $\theta \in \Theta$ que representa a constante normalizadora da densidade $a$ posteriori, portanto, é uma função somente dos dados $D$ observados. É comum se adotar a notação de proporcionalidade para representar a densidade a posteriori, 
como realizado neste estudo, dada pela equação 6 .

$\pi(\theta \mid \mathbf{D}) \propto L(\theta \mid \mathbf{D}) \pi_{0}(\theta)$

\section{A função de verossimilhança}

Para escrever a função de verossimilhança dos modelos (Tabela 1) foi considerado que na equação (1) o erro de ajuste $\epsilon_{i}$ é uma variável aleatória independente e identicamente distribuída com distribuição Normal $N\left(0, \sigma^{2}\right)$. Desta forma a função de verossimilhança [7] para os dados pode ser escrita a seguir, sendo que $\tau=1 / \sigma^{2}>0$.

$L(\beta, \tau \mid D)=\left(\frac{\tau}{2 \pi}\right)^{n / 2} \exp \left\{-\frac{\tau}{2}(\mathbf{Y}-\eta(\mathbf{x} ; \beta))^{\prime}(\mathbf{Y}-\eta(\mathbf{x} ; \beta))\right\}$

\section{Densidade de probabilidade a priori empírica}

A abordagem bayesiana foi iniciada considerando densidade de probabilidade a priori empírica. Para considerar as restrições do tipo $\beta \in R$, em que $R=\left\{a_{i}<\beta_{i}<b_{i}, i=0, \ldots, p\right\}$, foi considerada densidades a priori conjugadas Normal-Gama Truncada para os parâmetros, considerando-se $\beta \mid \tau \propto \mathrm{NT}\left(\mu,(\tau P)^{-1}\right)$ e $\tau \propto G\left(v_{0}, \lambda_{0}\right)$. Assim tem-se [8].

$\pi_{1}(\boldsymbol{\beta} \mid \tau) \propto \frac{\tau^{1 / 2}}{T(\boldsymbol{\mu}, \boldsymbol{P})} \exp \left\{-\frac{\tau}{2}\left[(\boldsymbol{\beta}-\boldsymbol{\mu})^{\prime} \boldsymbol{P}(\boldsymbol{\beta}-\boldsymbol{\mu})\right]\right\} \boldsymbol{I}_{R}(\boldsymbol{\beta})$

Em [8] $\mu=\left(\mu_{0}, \mu_{1}\right)^{\prime}$ (para os modelos de Stoffels e Petterson) e $\mu=\left(\mu_{0}, \mu_{1}, \mu_{2}\right)^{\prime}$ (para o modelo de Prodan) é o vetor de hiperparâmetros de locação e $P$ é a matriz de precisão, ambos conhecidos e $T(\mu, P)$ é uma constante normalizadora que não precisa ser calculada quando se usa o algoritmo MCMC. $I_{R}(\beta)$ é uma função indicadora tal que $I_{R}(\beta)=1$ se $\beta \in R$ e $I_{R}(\beta)=0$ caso contrário. A densidade a priori para $\tau$ foi dada por [9].

$\pi_{2}(\tau) \propto \tau^{\nu_{0}-1} \exp \left\{-\lambda_{0} \tau\right\}$

Em (8) $v_{0}$ e $\lambda_{0}$ são hiperparâmetros também conhecidos. A priori conjunta foi dada por [10].

$\pi_{0}(\boldsymbol{\beta}, \tau)=\pi_{1}(\boldsymbol{\beta} \mid \tau) \pi_{2}(\tau)$

Neste trabalho foi adotada uma abordagem empírica para a determinação dos hiperparâmetros $\mu$ e $P$. Para isso foram considerados os percentis $\left(H_{p}\right.$, $\left.D A P_{p}\right)$ e $\left(H_{q}, D A P_{q}\right)$, com $p<q$, encontrados em dados históricos coletados anteriormente às observações da amostra $D$, os quais não ocorreram obrigatoriamente de forma pareada (neste trabalho considerou-se nas aplicações $p=25 \%$ e $q=75 \%$, mas outros percentis poderiam ter sido considerados sem perda de generalidade).

Assumindo que os modelos da Tabela I são válidos para esses percentis, os parâmetros da densidade a priori empírica foram obtidos como descrito na Tabela 2.

A matriz de precisão $P$ também foi encontrada de forma empírica considerando um coeficiente de variação constante $\mathrm{CV}=\gamma_{\mathrm{j}} / \mu_{\mathrm{j}}^{*}, \mathrm{j}=0,1,2$ (BROEMELING, 1985) garantido que a mesma expressa a confiança nos hiperparâmetros $\mu$. Desta forma obteve-se [I I].

TABELA 2 Parâmetros da densidade a priori empírica.

TABLE 2 Parameters of the empirical a priori densities.

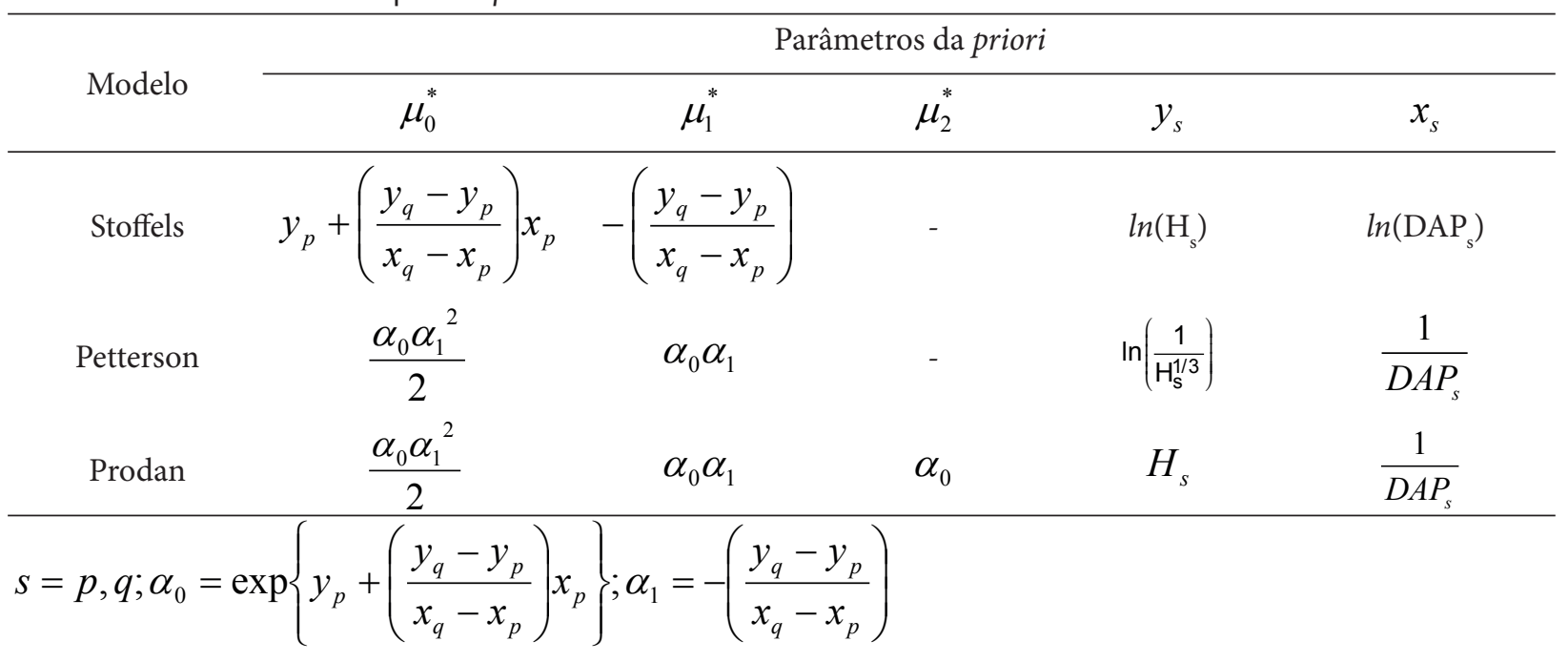


$P_{j, j}=\frac{1}{\gamma_{j}^{2}}=\frac{1}{\left(C V \mu_{j}^{*}\right)^{2}}, j=0,1,2$

Assumiu-se também que os coeficientes $\beta_{i}, i=0,1,2$ são independentes a priori, de forma que $P_{i, j}=0$ para $i \neq j,(i, j=0,1,2)$.

\section{A densidade de probabilidade a posteriori}

A função densidade de probabilidade a posteriori de $\beta$ e $\tau$ é dada por [12].

$\pi(\boldsymbol{\beta}, \tau \mid \boldsymbol{D}) \propto L(\boldsymbol{\beta}, \tau \mid \boldsymbol{D}) \pi_{0}(\boldsymbol{\beta}, \tau)$

Modelo de Stoffels

Para o modelo de Stoffels (THIERSCH et al., 20I3) (Tabela 1), a função densidade de probabilidade $a$ posteriori de $\beta$ e $\tau$ foi obtida substituindo-se [8] e [9] em [10], e [7] e [10] em [12]. Assim, tem-se [13], sendo, e é o estimador bayesiano irrestrito, dado por [14].

$\pi(\boldsymbol{\beta}, \tau \mid \boldsymbol{D}) \propto \tau^{n / 2+\nu_{0}} \exp \left\{-\frac{\tau}{2}\left[(\boldsymbol{\beta}-\widetilde{\boldsymbol{\beta}})^{\prime} \boldsymbol{V}(\boldsymbol{\beta}-\widetilde{\boldsymbol{\beta}})+\boldsymbol{W}+2 \lambda_{0}\right]\right\} \boldsymbol{I}_{R}(\boldsymbol{\beta})$

$\widetilde{\boldsymbol{\beta}}=\left(\boldsymbol{X}^{\prime} \boldsymbol{X}+\boldsymbol{P}\right)^{-1}\left(\boldsymbol{X}^{\prime} \boldsymbol{X} \widehat{\boldsymbol{\beta}}+\boldsymbol{P} \boldsymbol{\mu}\right)$

O próximo passo foi elaborar um algoritmo amostrador de Gibbs para encontrar o estimador bayesiano de $\beta$ e $\tau$. Foi adotada a seguinte notação [15] e [ I6], sendo $\Lambda(\beta)=(\beta-\widetilde{\beta})^{\prime} V(\beta-\widetilde{\beta})+W$.

$$
\begin{aligned}
& \boldsymbol{\beta} \mid \tau, \boldsymbol{D} \propto \mathrm{NT}\left(\widetilde{\boldsymbol{\beta}},(\tau \boldsymbol{V})^{-1}\right) \\
& \tau \mid \boldsymbol{\beta}, \boldsymbol{D} \propto \mathrm{G}\left(n / 2+\nu_{0}+1, \boldsymbol{\Lambda}(\boldsymbol{\beta})+2 \lambda_{0}\right)
\end{aligned}
$$

O algoritmo amostrador de Gibbs utilizado para gerar amostras da densidade a posteriori conjunta $\pi(\beta, \tau \mid D)$ é dado a seguir:

I. Faça $j=0$ e considere a condição inicial $\tau^{(0)}$.

2. Gere $\beta^{(j+1)}$ de $\beta^{(j+1)} \mid \tau^{(j), D} \propto N T\left(\tilde{\beta},\left(\tau^{(j)} V\right)^{-1}\right)$.

3. Gere $\tau^{(j+1)}$ de $\tau^{(j+1)} \mid \beta^{(j)}, D \propto G\left(n / 2+v_{0}+1, \Lambda\left(\beta^{(j)}\right)+2 \lambda_{0}\right)$.

4. Faça e repita os passos (2) e (4) até atingir a convergência.

Modelos de Prodan e Petterson

Para os modelos de Prodan e Petterson (Tabela 1), a função densidade a posteriori foi obtida substituindo na equação [10] as equações [8] e [9], e [10] e [7] na equação [12].
Em seguida foi elaborado um algoritmo de simulação de Monte Carlo em Cadeia de Markov (MCMC) para encontrar o estimador Bayesiano de $\beta$ e $\tau$. As funções densidade condicionais usadas no algoritmo de MCMC são dadas por [17] e [18], sendo

$$
\begin{aligned}
& \Psi(\beta, \tau)=\mathrm{L}(\beta, \tau \mid \mathrm{D}) \mathrm{NT}\left(\beta ; \beta_{0},(\tau \mathrm{P})^{-1}\right) . \\
& \boldsymbol{\beta} \mid \tau, \boldsymbol{D} \propto L(\boldsymbol{\beta}, \tau \mid \boldsymbol{D}) \mathrm{NT}\left(\boldsymbol{\beta} ; \boldsymbol{\beta}_{0},(\tau \boldsymbol{P})^{-1}\right) \\
& \tau \mid \boldsymbol{\beta}, \boldsymbol{D} \propto \Psi(\boldsymbol{\beta}, \tau) \mathrm{G}\left(\tau ; \nu_{0}, \lambda_{0}\right)
\end{aligned}
$$

O algoritmo MCMC (Metrópolis-Hasting) utilizado para gerar amostras da densidade a posteriori conjunta é dado por:

I. Faça $j=0$ e considere a condição inicial, $\tau^{(0)}$.

2. Gere $\beta^{(j+1)}$ de $\beta^{(j+1)} \mid \tau^{(j)}, \mathrm{D} \propto N T\left(\beta ; \beta_{0},\left(\tau^{(j) P}\right)^{-1}\right)$.

3. Aceite $\beta^{(j+1)}$ com probabilidade: $\alpha\left(\boldsymbol{\beta}^{(j)}, \boldsymbol{\beta}^{(j+1)}\right)=\min \left\{1, L\left(\boldsymbol{\beta}^{(j+1)}, \tau^{(j)} \mid \boldsymbol{D}\right) / L\left(\boldsymbol{\beta}^{(j)}, \tau^{(j)} \mid \boldsymbol{D}\right)\right\}$

4. Gere $\tau^{(j+1)} \propto G\left(\tau ; v_{0}, \lambda_{0}\right)$.

5. Aceite $\tau^{(j+1)}$ com probabilidade: $\alpha\left(\tau^{(j)}, \tau^{(j+1)}\right)=\min \left\{1, \Psi\left(\boldsymbol{\beta}^{(j+1)}, \tau^{(j+1)} \mid \boldsymbol{D}\right) / \Psi\left(\boldsymbol{\beta}^{(j+1)}, \tau^{(j)} \mid \boldsymbol{D}\right)\right\}$

6. Faça $j \leftarrow j+1$ e repita os passos (2)-(6) até a convergência ser atingida.

Para encontrar as estimativas bayesianas obtidas via simulação de Monte Carlo foi adotado o procedimento sugerido em Gilks (GILKS et al, 1998). Considerou-se um período de aquecimento (burn-in) de $50 \%$ das iterações iniciais. Os passos do algoritmo foram repetidos até que houvesse evidências de convergência, monitorada graficamente e com o critério de Geweke (GEWEKE, 1992). Então, uma amostra foi selecionada a cada 10 iterações. Logo, definindo $\theta^{(j)}=\left\{\beta_{0}^{(j)}, \beta_{1}^{(j)}, \tau^{(j)}\right\} . j=1, \ldots, M$ para os modelos de Stoffels e Petterson e $\theta^{(j)}=\left\{\beta_{0}^{(j)}, \beta_{1}^{(j)}, \beta_{2}^{(j)}, \tau^{(j)}\right\} \cdot j=1, \ldots, M$ para $\circ$ modelo de Prodan, a amostra resultante do algoritmo, os estimadores bayesianos obtidos via simulação de Monte Carlo foram dados pela equação 19.

$\widehat{g}\left(\theta_{s}^{M C}\right)=\frac{1}{M} \sum_{j=1}^{M} g\left(\theta_{s}^{(j)}\right)$

E os intervalos de credibilidade $\left[\hat{\theta}_{\mathrm{s}}^{\mathrm{MC}}(\alpha), \hat{\theta}_{\mathrm{s}}^{\mathrm{MC}}(1-\alpha)\right]$ foram estimados com base nos percentis da amostra, dados por [20].

$P\left[\widehat{\theta}_{s}^{M C}(\alpha) \leq \theta_{s}^{M C} \leq \widehat{\theta}_{s}^{M C}(1-\alpha)\right]=1-\alpha$

\section{RESULTADOS E DISCUSSÃO}

As estimativas bayesianas foram obtidas via simulação de Monte Carlo em Cadeia de Markov (MCMC). 0 
algoritmo gerou uma amostra de tamanho 40.000, da qual foram descartadas as primeiras 20.000 (burn in). Selecionou-se da segunda parte um valor a cada 10 gerados (ou seja, 20.00I, 20.010, 20.020,...,40.000), perfazendo, assim, uma amostra de tamanho 2.000 , que foi utilizada para o cálculo das estimativas bayesianas via MCMC.

Um sumário das estimativas bayesianas e clássicas pode ser observado na Tabela 3. Pode-se verificar nessa tabela que os resultados obtidos utilizando a densidade de probabilidade a priori empírica foram sempre coerentes biologicamente. Todos os resultados respeitaram as restrições impostas biologicamente, $\beta_{i}>0, i=0,1,2$. Os intervalos de credibilidade bayesianos ICred(95\%) revelaram a precisão dessas estimativas. Nota-se que para os modelos de Petterson e Stoffels na parcela $A$, os estimadores de mínimos quadrados (MQ) resultaram em valores positivos e essas estimativas co- incidem com as estimativas bayesianas, mostrando que houve consistência da abordagem bayesiana proposta com a de $M Q$, quando esta última foi factível.

Esse resultado também pode ser visto nas Figuras I e 2 e na Tabela 3, onde os parâmetros estimados positivos resultaram em curvas estimadas crescentes em todos os casos bayesianos, ao contrário do que ocorre com as curvas estimadas pelo método clássico, que em alguns casos (Figura I- A3 e Figura 2 - BI, B2, B3) geraram parâmetros negativos, violando, portanto, as restriçõos biológicas impostas aos parâmetros e inviabilizando seu uso.

\section{CONCLUSÕES}

A abordagem bayesiana empírica proposta em conjunto com a técnica de solução via MCMC mos-

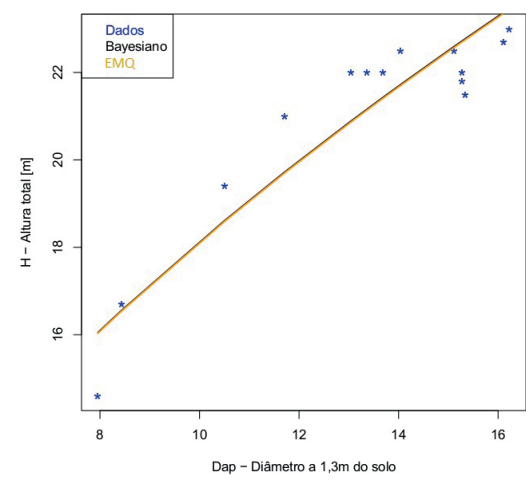

(A1)

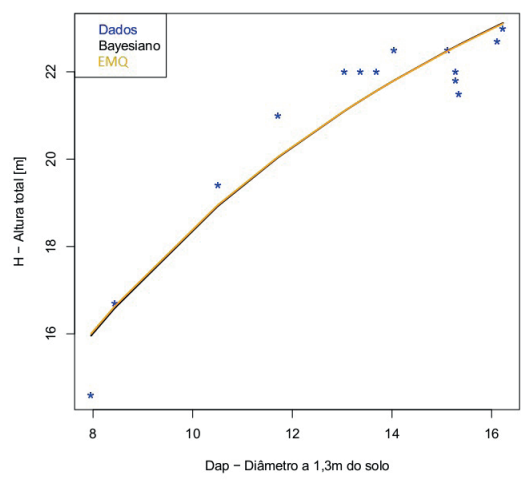

(A2)

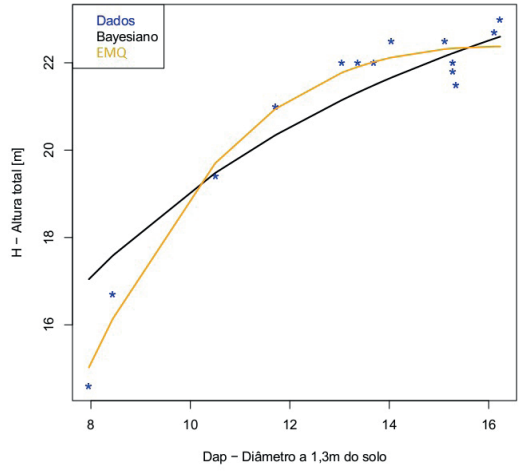

(A3)

FIGURA 1 Ajuste dos modelos hipsométricos de Stoffels (A1), Petterson (A2) e Prodan (A3) para a parcela A. FIGURE 1 Fitted hypsometric's model of Stoffels (A1), Petterson (A2) and Prodan (A3) for the sample plot A.

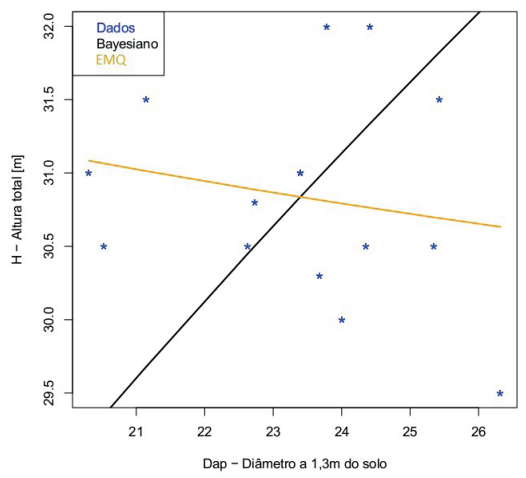

(B1)

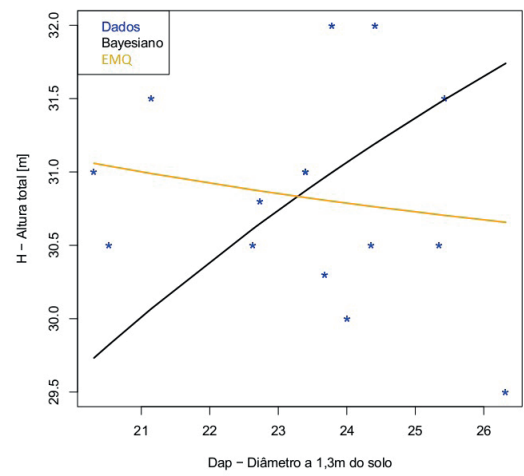

(B2)

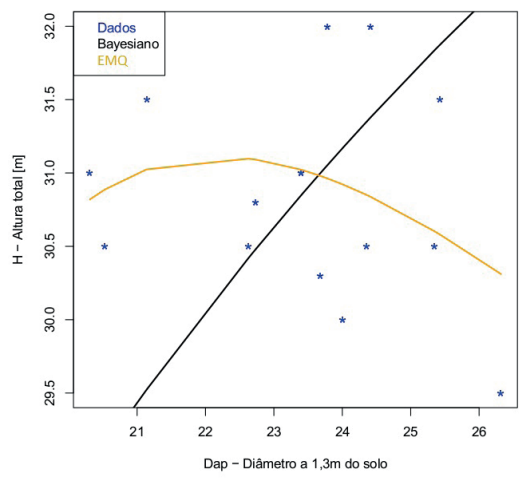

(B3)

FIGURA 2 Ajuste dos modelos hipsométricos de Stoffels (B1), Petterson (B2) e Prodan (B3) para a parcela B. FIGURE 2 Fitted hypsometric's model of Stoffels (B1), Petterson (B2) and Prodan (B3) for the sample plot B. 
TABELA 3 Estimativas bayesianas e de mínimos quadrados.

TABLE 3 Bayesian estimates and estimates of the minimum square.

\begin{tabular}{|c|c|c|c|c|c|c|}
\hline \multirow{2}{*}{ Parcela } & \multirow{2}{*}{\multicolumn{2}{|c|}{ Modelo/ Parâmetros }} & \multicolumn{2}{|c|}{ Estimativas Bayesianas } & \multicolumn{2}{|c|}{ EMQ } \\
\hline & & & Média & ICred(95\%) & Média & $\mathrm{IC}(95 \%)$ \\
\hline \multirow{7}{*}{ A } & \multirow{2}{*}{1} & $\beta_{0}$ & 1,6690 & $(1,6226 ; 1,7145)$ & 1,6694 & $(1,6647 ; 1,6737)$ \\
\hline & & $\beta_{1}$ & 0,5330 & $(0,5144 ; 0,5506)$ & 0,5329 & $(0,5311 ; 0,5347)$ \\
\hline & \multirow{2}{*}{2} & $\beta_{0}$ & 0,3066 & $(0,3023 ; 0,3108)$ & 0,3071 & $(0,3043 ; 0,3099)$ \\
\hline & & $\beta_{1}$ & 0,7211 & $(0,6668 ; 0,7772)$ & 0,7143 & $(0,6790 ; 0,7506)$ \\
\hline & \multirow{3}{*}{3} & $\beta_{0}$ & 0,4718 & $(0,3979 ; 0,5443)$ & 5,3032 & $(4,9980 ; 5,5802)$ \\
\hline & & $\beta_{1}$ & 0,1367 & $(0,1222 ; 0,1513)$ & $-0,6514$ & $(-0,6983 ;-0,5981)$ \\
\hline & & $\beta_{2}$ & 0,0340 & $(0,0329 ; 0,0352)$ & 0,0647 & $(0,0625 ; 0,0667)$ \\
\hline \multirow{7}{*}{ B } & \multirow{2}{*}{1} & $\beta_{0}$ & 2,2368 & $(2,2136 ; 2,2598)$ & 3,6065 & $(3,6028 ; 3,6102)$ \\
\hline & & $\beta_{1}$ & 0,3781 & $(0,3705 ; 0,3857)$ & $-0,0564$ & $(-0,0576 ;-0,0552)$ \\
\hline & \multirow{2}{*}{2} & $\beta_{0}$ & 0,2923 & $(0,2901 ; 0,2945)$ & 0,3242 & $(0,3202 ; 0,3282)$ \\
\hline & & $\beta_{1}$ & 0,6186 & $(0,5668 ; 0,6711)$ & $-0,1233$ & $(-0,2164 ;-0,0323)$ \\
\hline & \multirow{3}{*}{3} & $\beta_{0}$ & 1,5971 & $(1,2735 ; 1,9061)$ & 17,0885 & $(9,9086 ; 24,9146)$ \\
\hline & & $\beta_{1}$ & 0,1727 & $(0,1459 ; 0,2002)$ & $-1,5391$ & $(-2,2287 ;-0,8941)$ \\
\hline & & $\beta_{2}$ & 0,0221 & $(0,0209 ; 0,0233)$ & 0,0668 & $(0,0527 ; 0,0818)$ \\
\hline
\end{tabular}

trou-se vantajosa na consideração das restrições dos parâmetros do modelo. As estimativas bayesianas respeitaram os limites impostos aos parâmetros para todos os modelos e conjuntos de dados analisados, sendo que, o mesmo não ocorreu com as estimativas obtidas pelo método de mínimos quadrados.

A modelagem bayesiana proposta mostrou-se recomendável como técnica alternativa precisa e eficiente para a estimação dos parâmetros das relações hipsométricas.

\section{REFERÊNCIAS}

BROEMELING, L. D. Bayesian inference in statistical analysis. New York: Marcel Dekker, 1985. 545p.

CURTIS, R. O. Height diameter and height diameter age equations for second growth Douglas-fir. Forest Science, v. I3, n. 4 , p. $365-375.1967$.

GEWEKE, J. Evaluating the accuracy of sampling-based approaches to calculating posterior moments. Clarendon Press, Oxford, UK, 1992. In Bayesian Statistics 4.
PRODAN, M. Holzmesslehre. Frankfurt am Main: J. D. Sauerländer's Verlag. p. 44. 1965.

PRODAN, M. Forest biometrics. Oxford : Pergamon Press, 447p. 1968.

RIBEIRO, A.; FERRAZ, A. C.; MELLO, J. M.; FERREIRA, M. Z.; LISBOA, P. M. M.; SCOLFORO, J. R. S. Estratégias e metodologias de ajuste de modelos hipsométricos em plantios de Eucalyptus sp. Cerne, Lavras, v. I6, n. I, p. 22-31, 2010.

SOARES, T. S.; SCOLFORO, J. R. S.; FERREIRA, S. O.; MELLO, J. M. Uso de diferentes alternativas para viabilizar a relação hipsométrica no povoamento florestal. Revista Árvore, Viçosa, MG, v. 28, n. 6, p. 845-854, 2004.

STOFFELS, A. Die Genauikeit der Bestimmung der Stammzahl pro Hektar durch Messung von Stammabständen. Forstwissenschaft Centralblat, n. 74. p. 21 I-219. 1955.

THIERSCH, C. R.; ANDRADE, M. G. ; MOREIRA, M. F. B.; LOIBEL, S. Estimativa da relação hipsométrica em clones de Eucalyptus sp. com o modelo de curtis ajustado por métodos bayesianos empíricos. Revista árvore, v. 37, n. I, p. 0I-08. 2013. 